The International Journal Of Engineering And Science (IJES)

|| Volume || 5 || Issue || 11 || Pages || PP 01-14 || 2016 ||

ISSN (e): $2319-1813 \operatorname{ISSN}(\mathrm{p}): 2319-1805$

\title{
Fall Risk Assessment in Older People
}

\author{
Maryam Ghahramani, Fazel Naghdy, David Stirling, Golshah Naghdy, \\ Jan Potter
}

\begin{abstract}
-
Almost one-third of older people experience falls caused by medical factors and/or frailty. Falls may lead to devastating results. The rapid increase in life expectancy has made fall-detection and analysis an important issue. In order to identify trends and the state of the art in fall risk and balance assessment in older people, this paper carries out a review of the literature in this field. The focus has been on works that studied various assessment tools developed for fall risk assessment in people above 65 years old. The reviewed papers are categorized into two large groups of Clinical Fall Risk Assessment Methods and Quantitative Fall Risk Analysis Methods. For the studies in the first group, in addition to the contribution and limitations of each cited work, the validity and reliability factors are highlighted. The majority of the works reported in the second group are still in the development stage and have not been applied in real clinical applications. Hence, issues such as sensitivity and specificity are not considered. The review reveals that despite the high number of studies, there is no consistent worldwide standard for fall risk and balance disorder analysis and assessment. Many of the methods proposed have not been validated for large groups of subjects and the results are generalized without reliable evidence. High cost, complexity, and length of the procedure are the major drawbacks of many of the proposed methods. The frailty of older people can make routine gait analysis challenging. The results of the analysis of the current literature reveal a lack of any comprehensive, objective and accurate method.
\end{abstract}

Date of Submission: 25 September $2016 \longrightarrow$ Date of Accepted: 10 November 2016

\section{INTRODUCTION}

A fall is defined as a swift and unintentional change in the position leading to the descent of a person to a lower surface or level such as the ground[1]. A fall is caused when one's balance is disturbed. Balance has the definition of the ability to maintain equilibrium by keeping the centre of gravity over the base of support[2]. The human body usually reacts by different postural adjustments to keep balance but the compensation reaction for keeping balance among the elderly is usually delayed or not sufficient and this leads to a fall[3][4][5].

Balance disorders can be triggered by two groups of factors: intrinsic factors caused by inherent physical deficiencies such as visual impairment and postural hypotension, and extrinsic factors brought about by the environment and surrounding conditions such as a slippery floor. The intrinsic factors are associated with the effect of aging on the body [6], [1]. Many studies have focused on identifying the intrinsic fall factors. Some factors such as dizziness caused by medicine, loss of balance, syncope, postural instability, visual problems, neurological conditions, muscle weakness caused by natural deterioration, and sensorimotor deficiencies are identified as possible intrinsic fall factors [7].

Worldwide, approximately $30 \%$ of people above 65 years of age fall each year and this figure increases to $40 \%$ for people older than 80 [8]. Those who have fallen once are at risk of further falls and $52 \%$ of them fall again in the following 12 months [9]. Falls have the highest percentage of injury-related deaths [10]. The mortality rate of falls increases dramatically with age and $70 \%$ of accidental deaths in persons above 75 are caused by falling [11].Falling is the cause of $10 \%$ of the elderly being rushed to a hospital, $6 \%$ of which will require further hospitalization[12].

The consequences of a fall can be both physical, including fractures and other injuries, as well as social such as isolation, loss of confidence and depression [13], [14], [15], and[16].

It is obvious that accurate methods of measuring balance and physical activity in older people are critical as functional status is an important factor affecting the quality of life[17]. It is essential to record and classify the movement of an individual to specify his/her state of functional ability and overall level of mobility[16].

This paper presents a review of the methods proposed in the literature for balance, fall risk detection and mobility assessment in older people. Using a number of relevant keywords including "fall risk analysis for the elderly", "fall detection in older people", "elderly gait analysis", "older people activity recognition", "aged people movement recognition", and " balance analysis in aged people", more than 150 papers were identified and reviewed. 


\section{MeTHODS}

The papers identified were processed through a rigorous and thorough approach. In the first stage, the title and abstract of each paper were examined to understand the hypothesis and aim of the study.

Then among the works identified papers with sound and relevant hypotheses, and high citations and which satisfied the following criteria were selected for review:

a) The aim, hypothesis and objectives of the paper were clearly described.

b) The main aim of the study was to analyse fall factors among the elderly or test one or several fall risk assessment tools.

c) For the clinical papers, the focus of the paper was on testing the reliability and validity factors of a special fall risk analysis tool such as specificity, sensitivity, etc.

d) The method deployed in the paper was specifically applied to older people.

e) The deployed intervention and methodology in the paper were comprehensively explained.

f) The mathematical and statistical modelling and analyses used in the paper were appropriate, correct and accurate.

The selected papers were subsequently structured as illustrated in Figure 1 (appendix 1) according to their foci and approach.

In this review, the focus will be on both the clinical and quantitative methods used in fall risk assessment.

Fall Risk Assessment:

\section{Clinical-Based Methods}

\subsubsection{Functional Assessment Methods}

Functional assessment methods are very common in the clinical environment as they are low cost, easy to deploy, fast, and often do not need any special expertise[18]. They are usually scored on a three to five scale or based on timing, i.e. the duration that each subject can keep balance in a particular balance test [19]. The most important functional assessment methods are: 1-the tandem stance,2-theone leg stance, 3-the functional reach Test,4- the sit to stand test, 5-the clinical test of sensory interaction for balance (CTSIB), 6-the timed get up and go test (The TUG), 7- the 6 minute walking test (6MWT), 8- the dynamic gait index, 9-the Berg balance scale (BBS)test, 10-the Tinetti gait and balance test, and11-the Barthel index.

\subsubsection{Tandem Stance Test (Romberg Test)}

In the tandem stance test or the Romberg test, named after Moritz Heinrich Romberg, the patient is asked to stand with their feet together and arms by their side with open eyes for about 10 seconds. Then, the patient is asked to repeat the test with their eyes closed. The patients are categorized as able and unable based on their ability to do the test [20]. Murphy et al. [21] find a sensitivity of 55\% and a specificity of $94 \%$ for the tandem stance tests. In another study by Agrawal et al.,[22]a modified Romberg test is used. In the modified version, subjects are asked to conduct the Romberg tests on firm and compliant support surfaces and their performance is assessed based on timing. They observe an inverse relationship between time and failure and age. For the subjects with the time to failure of less than 20 seconds, there is a greater than three-fold increase in the odds of falling. Subjects at age 60-69 cross the threshold of 20-seconds. In another study the inter-rater reliability of $99 \%$ and test-retest reliability of $90 \%$ are found [23].

\subsubsection{The One-Leg Stance Test}

In this test, the subject's ability to maintain balance while standing on one leg is assessed and the performance is scored based on timing (0-30 seconds) [24]. The test is also repeated with the subject blindfolded. Some believe that the single-leg stance is the most important test for sway and balance analysis as $20 \%$ $40 \%$ of the time walking is done on one foot support [25]. For the one-leg stance test with eyes open the interrater reliability of $99 \%$ and test-retest reliability of $90 \%$, and for the one-leg stance test with eyes closed the inter-rater reliability of $99 \%$ and the test-retest reliability of $74 \%$ are reported [23]. As a drawback of the approach, the majority of older people cannot perform the one-leg stance test as they lose their balance immediately or they do not agree to do the test because they are afraid of falling [26].

\subsubsection{The Functional Reach Test}

According to [27] the functional reach test is used to measure a subject's ability to lean forward as much as possible without moving the lower body or losing balance. Duncan et al. [28] find the test useful and effective on predicting the risk of fall. It is believed that the test shows the subject's ability to move his/her centre of gravity (the COG) toward the edge of support[24]. Recently, however, it has been observed that due to compensatory strategies in reach, the COG displacement is not completely correlated with the functional reach distance[29].Behrman et al. [29]believe that the functional Reach Test, with a specific threshold, is not sensitive enough to identify patients at risk of fall. Those identified as being at risk do, indeed, have a high likelihood of 
having a fall, but there are others who are also at high risk of falling who may not be identified by the test. An inter-rater reliability of $96 \%$ and test-retest reliability of $86 \%$ have been found in the study conducted in[23]. In another study, a sensitivity of $73 \%$ and a specificity of $88 \%$ are recorded for the functional reach test with eyes open [21]

\subsubsection{Sit to stand Test (STST)}

In this test, the subject is asked to fully stand up and then sit down as fast as possible 5 times or 10 times without the use of the hands and arms. The test is scored based on timing [30]. In a study conducted by McRaeet al.[31] it is observed that fallers have greater sit to stand test time compared to non-fallers. Franchignoni et al.[23] report an inter-rater reliability of $98 \%$ and test-retest reliability of $92 \%$.

\subsubsection{The Clinical Test of Sensory Interaction for Balance( CTSIB)}

This method was first described by Shumway-Cook and Horak [32]. The patients are asked to stand with their hands at their sides, feet together first on a firm surface then a soft surface such as foam with eyes open, eyes closed, and a visual conflict dome. Later, in the modified version, the tests with visual conflict dome were eliminated [33]. In this test, the subject's ability to use visual, somatosensory and vestibular input for maintenance of balance while standing is assessed [34]. Each Test is terminated when the subject's arms or feet change position. The total score is the sum of the time of each test. In a study conducted by Di Fabio and Anacker [35] using CTSIB for subjects over 81 years of age, $83 \%$ of the elderly subjects with no history of fall and $80 \%$ of the fallers are classified correctly. For subjects younger than $82,95 \%$ of the non-fallers and $67 \%$ of the fallers are classified correctly. The CTSIB is found to be a sensitive and specific measure of recurrent falls in older people living in the community, but only when the pattern of sensory-interaction abnormality during stance on foam is used to determine fall status. Cohen et al. [33] find an excellent inter-rater reliability of $99 \%$ for the CTSIB.

\subsubsection{The Timed Get Up and Go Test (TUG)}

The timed up and go test is one of the quickest and easiest gait and balance assessment methods [36]. It is the modified version of the get up and go test that was first introduced by Mathias and associates [37].In this test the subject is asked to get up from a chair, walk for about 3 meters, turn around, walk back and sit down on the chair. Shumway and colleagues find the timed TUG successful in predicting the elderly subjects at risk of fall[38]. Lunndin-Olsson et al.[39]find that a modified TUG test, made more sensitive in predicting the risk of fall by adding peripheral tasks such as holding a glass of water, are quite effective. The TUG test has a combination of several important mobility skills like walking, turning, sit-to -stand, and stand-to-sit transitions which make it an effective fall risk assessment method [40]. It is not possible; however, to specify which balance subcomponent is impaired [41].A test-retest reliability of 56\% is reported in a study by Rockwood et al. [42].

\subsubsection{The 6-Minute Walking Test (6MWT)}

The 6-minute walking test (6MWT) first developed by Balke is proposed to evaluate functional capacity [43]. The 6MWT is assessed based on the distance the subject walks over six minutes on a hard and flat surface (6MWD). The subject is expected to walk as far as possible in six minutes. Although many walking tests with different durations have been tested, the 6MWTis reported to be easier to conduct and retest compared to longer timed tests. It is also reported that walking tests less than 4 minutes are not sensitive enough [44]. According to [45] the 6MWD has a significant correlation with age. Harada et al. [46]find 6MWD significantly largerfor active elderly compared to inactive older adults. They also find a test-retest reliability of $95 \%$ for the 6MWT.

\subsubsection{The Dynamic Gait Index (DGI)}

The dynamic gait index consists of 8 walking tests: steady-state walking, walking while changing gait speed, walking while moving the head vertically and horizontally, walking while stepping over and around an obstacle, turning during walking, and stair climbing. The subject's performance is scored between $0-3$ on each test, yielding an overall score out of 24[47]. Shumway-Cook et al.[47]find the inter-rater of 96\% and test-retest reliability of $98 \%$ but the threshold score of 19 or less on the DGI correctly classifies $59 \%$ of fallers. In another study by Herman et al.[48]it is reported that fallers have lower DGI compared to non-fallers. They report good sensitivity for detecting fallers (91\%), but poor specificity $(3 \%)$ in this cohort of healthy older adults with the threshold of 19. It is observed that higher thresholds improve specificity, but decrease sensitivity; and no threshold results in both good $(\geq 80 \%)$ sensitivity and specificity. Also, the DGI suffers from the ceiling effects[49]. 


\subsubsection{Berg Balance Scale (BBS) Test}

The Berg balance scale (BBS) test was first introduced by Berg[18]. It consists of the following 14 activities:

1-Sitting to standing,

2-Standing unsupported,

3-Sitting with back unsupported, feet on floor,

4-Standing to sitting,

5-Transfer from an armed chair to a bench and back,

6-Standing unsupported with eyes closed,

7-Standing unsupported with feet together,

8- Reaching forward with outstretched arm while standing,

9-Picking up an object from the floor from a standing position,

10-Turning to look behind over left and right shoulders while standing,

11-Turning 360 degrees,

12-Placingalternate foot on step or stool while standing unsupported,

13-Standing on one leg,

14-Standing unsupported, one foot in front.

In the BBS method, participants are scored by a clinician for each task on a scale of $0-4$, with a final score out of 56 as the sum of the scores given for the tasks. It is assumed that ambulatory ability is determined by the quality of balance [50].

Stevenson et al. [50] define two thresholds based on the data from two separate projects consisting of 171 subjects aged above 65: 1) BBS Threshold of walking without any gait aid $(\geq 49 / 56), 2)$ BBS threshold of walking without a four-wheeled walker ( $\geq 43 / 56)$. In other words, 43-49 are the scores defined as the scores which the subject needs walking aid and 43 or less are the scores showing that the subject needs a four-wheeled walker.

Thorbahn et al. [51]use the BBS test to assess the risk of fall in older people. The data from a total of 66 subjects aged between 69 and 94 is utilized in the study. The overall assessment distinguishes between the subjects who are prone to falling and those who are not. The test, however, is not sensitive enough to predict the frequency of fall.

Maeda and Kato[52]observe that BBS is highly related to falls, as fallers have lower BBS scores than non-fallers. The BBS scale is easy to use, does not need any specific device, has excellent specificity (96\%) and inter-rater reliability $(88 \%)$ but the sensitivity is poor $(53 \%)$ and as it is subjectively scored by clinicians, inconsistency between two scores is very common[51][53]. In another study, the inter-rater reliability of 0.8 is reported[54].

\subsubsection{The Tinetti Gait and Balance Test}

The Tinetti gait and balance assessment test also known as "performance-oriented mobility assessment (POMA)" is one of the most common clinical balance assessment methods for use with the elderly[55][36]. The balance assessment part is scored based on the quality of the subject's performance on different balance tests such as sit to stand, sitting, standing with eyes closed, turning around, etc. The gait part is scored based on different information about the subject's walking, such as gait speed, gait width, gait height, etc. The balance part is scored out of 16 and the gait part is scored out of 12[56]. The Tinetti mobility test is reported to have good inter-rater and intra-rater reliability, excellent sensitivity and good specificity. Application of the Tinetti mobility test to subjects between 64 and85 years of age and a cut-off score of 20 results in a sensitivity of $78 \%$ and a specificity of 75\% [57]. The Tinetti test, however, has a ceiling effect and, like other clinical methods, is subjective and prone to human error [36]. In a study by Raiche [58], a specificity of 53\%and a sensitivity of $70 \%$ are reported for a threshold score of 36.

\subsubsection{System Assessment}

The system assessment methods aim at finding the underlying causes of balance disorders in order to facilitate treatment.[19]. The system assessment methods include the Physiological Profile assessment method andthe Balance Evaluation system Test.

\subsubsection{Physiological Profile Assessment (PPA)}

Many studies have shown that factors such as vision impairment, peripheral sensation, muscle strength, reaction time, and balance are significant fall factors with a reverse relationship with age. Based on these factors, a physiological profile assessment (PPA) is proposed by Lord and Menz [59]. Any impaired factor increases the risk of falling. In this method, several tests which are brief, easy to conduct, and feasible for older people are used. The tests are also low-tech and robust in order to be more practical in large population studies. 
Tests such as visual tests, tactile sensation, vibration sensation, peripheral sensation, muscle force, reaction time, and balance are used in order to score the risk of falling. Lord and his colleagues in three different studies conclude that PPA is a satisfactory and reliable measure of fall risk with a high sensitivity of $75 \%$ and a testretest reliability of 70\% [60][61][59], but the PPA cannot help direct treatment because the functional ability of a subject is dependent on other factors such as compensation, age, motivation, etc. as well as pathology[62].

\subsubsection{The Balance Evaluation System Test (BESTest)}

The balance evaluation system test (BESTest), first introduced by Horak et al, consists of 36 items, categorized as 6 groups of biomechanical constraints stability limits/verticality, anticipatory postural adjustments, postural responses, sensory orientation, and stability in gait[62]. In a study conducted by Horak et al. it is shown that the BESTest has excellent reliability and very good validity. It has an inter-rater reliability of $91 \%$ and can identify the nature of balance problems that can help in devising and deploying a specific treatment for a patient. On the other hand, it is not known for sure whether the sections of the BESTest can detect independent balance deficits, and whether it needs to be combined with other balance systems for better results [62]. It usually takes 30 minutes to conduct the test, though recently a shorter version of the test with the duration of 10 minutes has been developed [63].

\subsection{Fall Risk Assessment Tools at care settings}

Falls are common among older hospital inpatients and these typically lead to longer stays in a hospital. The methods used for elderly inpatients should be quick, easy to conduct and safe as the elderly inpatients are more fragile. There are many methods designed to predict falls for inpatients prone to fall. Since these methods mostly rely on self-reporting, they are not fully reliable as some fallers may not be able to report their fall and explain or remember the exact reason for their fall and circumstances surrounding it. It should be also considered that many of the patients at risk of fall may never fall and many falls may be accidental due to extrinsic factors such as a slippery floor, and etc.[64].

\subsubsection{The STRATIFY Tool}

The Saint Thomas's risk assessment tool or STRATIFY is based on five questions:

1-Is the patient hospitalized because of a fall?

2-Is the patient agitated?

3-Does the patient have vision impairment?

4-Does the patient need toileting frequently?

5-What is the state of the patient's ability and independence in transfer and mobility?

Each test is scored between 0-1[65].

Oliver et al. [65] conduct the STRATIFY tool in three consecutive phases to check its ability to correctly identify elderly hospital inpatients at risk of falling. They conclude that STRATIFY is a valid fall risk assessment method with clinically useful sensitivity and specificity.

An extensive study using STRATIFY has been undertaken in Belgium by several researchers in order to assess its potential for the prediction of falls [66]. Data from a large group of inpatients with the average age of 67 was obtained. The results show that the STRATIFY has a good sensitivity and high negative predictive value for patients younger than 75, with moderate to low sensitivity and high false-negative rates for patients aged 75 and older. The results also show that the STRATIFY approach has been a good fall predictor for patients 74 and younger but significantly less effective for patients above 75 .

This study has a number of limitations, however: 1- The prevention of patient falls by assistance rendered by nursing staff that may have biased these results. 2- The low probability of falls in some hospital units such as a surgical unit may have led to low positive predicted values. 3- As many patients are rushed to hospital after a fall in the evening and at night, this may have contributed to their agitation and consequently also biased the predicted outcomes. 4- A major deficiency of this method and all methods involving human interpretation is that it involves subjectivity which is inconsistent and prone to errors [65].

In a study by Papaionnou [67] with a risk score of 9 , sensitivity of $91 \%$ and specificity of $60 \%$ and inter-rater reliability of $78 \%$ are reported.

\subsection{2. $\quad$ Morse Fall Scale}

The Morse fall scale was first introduced by Morse et al. [64]. It is developed based on establishing a database from 100 patients who had a fall and 100 patients who did not have a fall. The database includes significant variables that differentiate between the fallers and the non-fallers. Morse Fall Scale consists of 5 factors: 
1- History of Fall(0 no falls, 15 previous falls)

2- Secondary Diagnosis ( 0 no, 15 if more than one medical diagnosis is listed)

3- Ambulatory Aid (0 none/bed-rest/nurse assist, 15 crutches/cane/walker, 30 furniture)

4- Intravenous Therapy ( 0 no, 20 if the patient has an intravenous apparatus)

5- Gait (0 normal gait, 1 weak gait, 2 impaired gait)

6- Mental Status (The patient is asked about their abilities, 0 if realistic and oriented to own ability, 15 if overestimating their ability or forgetful of their limitations)

Morse et al. [64] correctly classify $78 \%$ of the fallers and $83 \%$ of the non-fallers with a threshold of 45 . They also obtain aninter-rater reliability of $96 \%$. In another study by McCollum et al. [68] a sensitivity of 83 $\%$, a specificity of $68 \%$ and an inter-rater reliability of $98 \%$ are recorded.

A major problem with the Morse fall index is the great weight for previous falls as many of the patients at risk of fall may never have fallen and many fallers may have experienced accidental falls due to extrinsic factors such as a slippery floor, etc.[64].

\subsubsection{Downtown Index}

The downtown fall risk index includes 11 fall risk factors [69]. Each factor is scored 0-1 yielding to an overall score out of 11.A cut-off score of 3 is set up for high-risk falls.

Downtown fall risk factors are as follow:

1- History of fall ( 0 no, 1 yes)

2- Medications ( 0 none, 1 a list of special medications)

3- Sensory deficits (0 no, 1 Visual impairment/Hearing impairment/ Limb impairment )

4- Mental status ( 0 oriented, 1 confused)

5- Gait (0 normal/safe with aid/ unable, 1 unsafe)

In a study by Nyberg et al.[70]the Downton index is used to predict elderly stroke patients at risk of fall. They have reported a good sensitivity of $91 \%$ but a poor specificity of $27 \%$ for the Downton fall risk index.

In another study, the risk of fall for a group of older people in residential care facilities is predicted using the Downton index. Sensitivities ranging from 81 to $95 \%$ are recorded, with the highest value at 3 months, and specificity ranging from 35 to $40 \%[71]$.

\subsubsection{The Barthel Index}

The Barthel index of daily activity was first introduced by Dorothy Barthel. This is a measure of disability of patients with neuromuscular and musculoskeletal conditions receiving inpatient rehabilitation. Ten variables representing daily activities such as bathing, toilet use, mobility, grooming, etc. are listed. The subject is scored based on the quality of performance and the Barthel index is scored in steps of five points to give a maximum total score of 100. A widely adopted modification to the index includes a revised score range of 0-20 with better sensitivity [72].In the study conducted by Collin et al.[73] the validity of the Barthel index, with good reliability and validity in each task is demonstrated.

\section{Quantitative methods}

Over the last decade, a number of quantitative body balance and fall risk analysis methods producing objective and reliable outcomes have been proposed. Quantitative methods deploy advanced sensory systems and data collection and analysis to enhance the reliability and repeatability of the qualitative methods. In these methods, interventions such as the deployment of force platforms, inertial sensors, and reflective markers produce more accurate outcomes. Such studies, however, have not resulted in a widely accepted standard or method for fall risk assessment.

The quantitative fall risk assessment methods can be divided into two groups: static postural balance assessment and motion analysis methods.

\subsection{Static Postural Balance Analysis}

The static postural balance assessment methods measure postural sway in the static tests for balance analysis. Spirduso defines body sway as the individual's small body postural movements made to maintain balance. Body sway can be analysed by measuring the total displacement of the centre of mass over time [74]. In different studies, postural sway is defined as the amount of movement in different parts of the body, such as sacrum [75], waist [76] upper part of the body at the level of shoulders [77], trunk [26]or the whole body[78]. Most studies express sway as the displacement of the centre of Pressure (COP) or the centre of gravity (COG) [79]. 
3. The human body normally sways as a result of breathing or shifting body weight from one foot to another. Body sway can intensify as a result age or illness [80]. Various observations also show that the body sway increases after the age of 40[81]. Many studies conclude that there is an association between age and postural sway [76],[77] but no precise correlation between postural sway and ageing has yet been identified. A variety of equipment has been deployed to analyse body sway quantitatively. Sway magnetometry, Wright ataxia-meter, potentiometric displacement transducer are devices which record the sway pattern and sway distance of subjects at the wrist level[82],[83],[84]. Recently, force platforms and accelerometers have been used to analyse body sway more accurately [85].

\subsubsection{The potentiometric displacement transducer}

The potentiometric displacement transducer consists of an aluminium pad that is mounted around the subject's waist connected to a transducer. The transducer records the subject's anteroposterior displacement at the waist level [86]. This outdated approach, however, can only provide information about waist displacement in the anteroposterior direction under static conditions. It may not be safe to be used by the frail elderly because it adds extra weight for the subject to carry. [87].

\subsubsection{Wright Ataxia-meter}

The Wright ataxia-meter, first introduced by Wright, analyses anteroposterior sway by recording the number of rotations of a wheel, which is attached to the subject's waist by a string [88]. In a study conducted by Brocklehurst at al. [89] the Wright ataxia-meter is used to analyse skeletal deformities in the elderly and study their effect on postural sway. They observe that postural sway is correlated with the loss of height in the elderly.

\section{Static Force Platforms}

Static force platforms are used to measure the displacement of the centre of pressure of a subject while standing still [2].

Swanenburg et al.[90]conduct an extensive study of fall assessment in a large group of elderly people using portable force platforms. The standing tests are carried out with eyes open and eyes closed. They have also added cognitive tasks by asking the subjects to count backward in steps of seven to analyse the dual task balance tests using static force platforms. Despite many studies claiming that dual tasks add value to fall prediction [91]in the elderly, this study shows no increased value in the dual-task situation compared to that of a single task. Using static force platforms, they conclude that the medial-lateral sway in the elderly with a history of falls is significantly greater.

Haibach et al. [92]also assess postural sway using force platforms. One group of young adults and two groups of elderly; one group with a history of falls and the other without are placed in a simulated moving scene. The subjects are asked to stand still on the platform whilst fixating upon a focal point on a screen that presents a virtual reality view of a moving room, forcing them to sway and move. The simulated motion causes postural reactions in all subjects during the experiment. The study is successful in distinguishing young subjects from older subjects and older non-faller subjects from older faller subjects by using force platforms as elderly subjects sway more than younger people and fallers more than non-fallers. Static force platforms are reported to have good test-retest reliability [87].

\subsection{Motion Analysis Methods}

\subsubsection{Dynamic force platforms}

Force platforms play a major role in gait analysis. The unobtrusiveness of this method makes it suitable for gait analysis in older people as any additional barriers may affect their motion and walking. Dynamic force platforms are used to measure the displacement of the centre of pressure under dynamic conditions [87].

Kerrigan et al. [93] utilise a force platform and optical markers in order to analyse the walk of older people at both comfortable and faster speeds. Nine parameters related to the knee, hip and ankles such as flexion moment, power generation loading response and power absorption are found to be extremely different at comfortable walking speeds between fallers and non-fallers.Four of these parameters also remain significantly different at the faster-walking speeds. It is unclear whether the kinetic differences are the factors leading to falls or if they reduce the risk of falling. Force platforms have been reported to have a good test-retest reliability [87].

Verghese et al. measure quantitative gait factors using a computerized walkway [94]. The participants are followed up every 2-3 months and the number of their falls, their cause and the associated injuries are recorded. In this study, seven factors are confirmed as fall predictors. These factors include a $10 \mathrm{~cm} / \mathrm{s}$ unit decrease in gait speed, a $10 \mathrm{~cm}$ decrease of stride length, a $10 \%$ decrease in the swing phase, a $10 \%$ increase in the double-support phase, a $10 \%$ increase in stride length variability, a $10 \%$ increase in swing time variability. Participants with a gait speed of less than $70 \mathrm{~cm} / \mathrm{s}$ are shown to be more prone to fall compared to those with a 
gait speed of more than $100 \mathrm{~cm} / \mathrm{s}$. Verghese et al. note that each $10 \mathrm{~cm}$ decrease in gait speed is associated with a $7 \%$ increased risk of fall. Here, gait speed is considered as the most important factor in fall risk assessment.

In further work carried out by Kerrigan et al.[95], the gait of a group of healthy elderly people aged between 65 to 84 and a control group of young subjects between 18 and 36 is assessed using infrared reflective markers and two force plates. The study shows that peak hip extension is reduced in the elderly group in normal walking and has no change in fast walking. The reduction in hip extension is found to be associated with an increase in anterior pelvic tilt. It is also shown that reduced peak hip extension, increased anterior pelvic tilt, and reduced ankle plantarflexion and ankle power generation are age-related kinematic and kinetic gait changes persisting at both comfortable and fast walking speeds.

Winter et al. [96] use force platforms and optical passive markers to detect changes in the walking pattern of the elderly. The gait pattern changes caused by normal biological deterioration rather than any neural, muscular or skeletal disorder are investigated in fifteen healthy and fit elderly people. Twelve young adults are also recruited to undergo the test as the control group. Each subject is asked to walk at his/her natural velocity on a level walkway at least 10 times. The results obtained in this study indicate that:

- The walking velocity of older people is lower than younger people. The reduction in velocity is caused by an increase in stride length and double-support stance time (both feet touching the ground).

- The older and younger subjects do not show significant differences in their toe clearance (disconnecting the toe from the ground completely).

- The covariance between the hip and knee moments of force patterns is a little smaller in the elderly than in younger people.

- Elderly subjects have smaller push-off power and more flat-footed landing than younger people.

\subsubsection{Inertial Sensors}

Inertial motion capture is based on accelerometers and gyroscopes. Analysing the kinematics of rigid body motion requires estimation of translational and angular velocities and accelerations of the body centre. Inertial sensors or inertial measurement units (IMU) can dynamically measure these parameters, and consequently, they are widely deployed in biomechanics. The miniature size of these sensors makes them ideal for implementing small mobile embedded systems. Most of the body-mounted inertial sensors integrate more than one type of sensor to increase the accuracy of sensors or foot switches and goniometers. The acceleration of different body parts and the effect of gravity are measured by accelerometers. Gyroscopes are mainly used to measure the angular velocity. Magnetometers are often deployed to measure changes to orientation. Foot switches can recognise foot contact with the ground and goniometers are mainly utilized to measure changes in joint angles [13].Many studies use inertial sensors to successfully analyse the risk of fall among older people [97].

Najafi et al.[98][99] measure the times of sit to stand, stand to sit and their duration in elderly people with the use of an accelerometer. The actual trunk tilt corresponding to angle $\theta$ between perpendicular line and the thorax trunk of the subject and displacement of the gyroscope are measured (Figure 2). TD is defined as the time difference between beginning of tilt down to the end of tilt back during the stand to sit or sit to stand. Finally the mean of TD (M-TD), its standard deviation ( $\Delta$-TD) and the number of attempts to have a successful transition (S-Trs) are measured. The M-TD and $\Delta$-TD have larger values for individuals with high risk of fall than for those at low risk. These three parameters are proposed as suitable indicators in fall risk assessment.
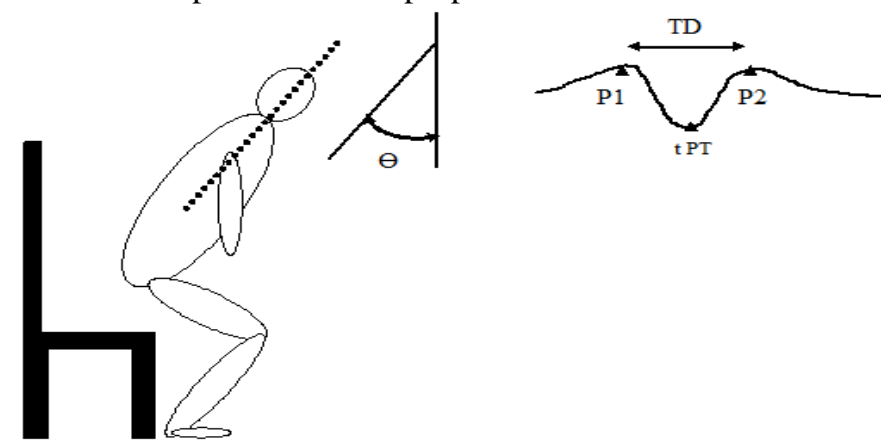

Fig. 2(a) Angle $\theta$ between subject's trunk and vertical surface (b) During a sit to stand test PT is detected from the pattern of $\sin \theta, \mathrm{P} 1$ is the beginning of tilt down and P2 is until the end of tilt back during a sit-stand (vs. stand-sit) transition. P1 and P2 are estimated by detecting the maximum peaks before and after $\mathrm{PT}$

Yack and Berger [99] analyse trunk acceleration with body-worn accelerometers. An accelerometer is mounted over the subject's uppers pine. They are then asked to walk for approximately 40 seconds. Using a finite Fourier series, the data is analysed, and in-phase and out-of- phase components of movement are 
identified. An index of smoothness defined as a ratio of even harmonics divided by odd harmonics is also derived. Stable subjects in both older and younger groups indicate higher indices of smoothness compared to unstable subjects. It is thus concluded that stability problems are proportional to the inability to control the trunk. As stable and unstable elderly subjects are determined by questionnaires and clinical tests, the method is not completely reliable.

Menz and Lord further [100] analyse acceleration signals by using inertial sensors mounted on the body of elderly subjects. The subject's accelerations are measured using two accelerometers, one attached to the level of the sacrum and another attached to the head. Based on the results, the subjects are divided into three groups: low-risk, moderate-risk and high-risk fallers. Menz and Lord conclude that the high-risk fallers have lower velocity and smaller step length compared to low-risk fallers on smooth surfaces and compared to both low-risk and moderate-risk fallers on irregular surfaces. The high-risk fallers are found to have a smaller harmonic ratio of head and pelvis. The main conclusion is that the elderly at risk of fall have difficulty controlling trunk motion, especially on irregular surfaces.

Mariani et al. [101] utilize two tri-axial accelerometers attached to the feet and an optical system as the reference. The tests are applied to ten younger and ten older people in which the four parameters of stride length, stride velocity, foot clearance, and turning angle are measured. Stride length is defined as the distance between foot-flat positions. Foot clearance is defined as the maximum height of the foot coming up while walking. Stride velocity is the mean value of foot velocity during each gait, and the turning angle is the azimuth change in a gait cycle (Figure 3). The results show no significant difference in the mean values of stride length and stride velocity between the two groups. The most distinct parameter found is foot clearance, which is significantly different between the older and the younger people. It is also observed that the elderly have larger stride velocity on a smooth path and smaller stride velocity while turning. This may happen when the elderly try to compensate by quicker strides on smooth paths, particularly as they have problems in controlling their strides while turning.
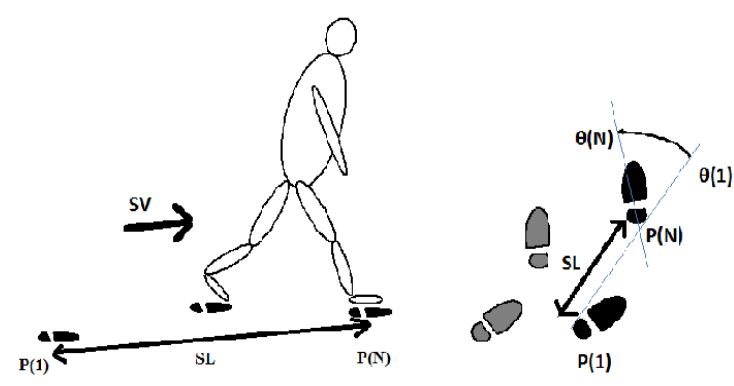

Fig. 33 D foot position $(\mathrm{P})$ and azimuth $(\theta)$ : stride length (SL), stride velocity (SV), and turning angle (TA).

\section{DISCUSSION AND CONCLUSION}

A summary of the characteristics of clinical methods including Functional, Systematic and Fall Risk Analysis for Elderly Inpatients are shown in Tables I-III. The parameters provided in these tables are measured during their deployment of these methods in a clinical environment. Such data is not yet available for quantitative balance and fall risk analysis methods as most of them are laboratory based and not used in clinical applications.

The clinical methods are often easy to conduct and assess. They do not need special equipment and hence can be carried out anywhere. Usually, the clinicians who run the tests do not need much training.

There are, however, many drawbacks and deficiencies associated with clinical methods. Many of them are assessed based on the distance walked by the patient or the length of time it takes to walk but none of the parameters of distance or time provide any information on the exact biomechanical impairment that leads to longer walking time or shorter walking distance. For example, the timed get up and go test consists of many balance subcomponents such as standing up, walking, turning and sitting down, but measuring the timing of this test does not give any indication of which balance sub-component has significantly affected the assessment. In the 6MWT, the performance of the subject is assessed based only on the distance walked in 6 minutes, not on the pattern of the subject's gait.

Some tests such as standing on one leg intimidate older people, especially those with a history of falls to the extent that they refuse to do the test. This, however, does not mean that the subject is not capable of carrying out the test.

There are tools that analyse different balance abilities such as the Berg balance scale and the Tinetti scale. They consist of different balance tests which all simulate the body postures that usually result to falls in older people. In spite of their relevance, these methods are subjective in their assessment approach. In each test, 
the performance of the subject is scored by a clinician with a value between 0 and 4 . The fact that the tests are scored subjectively through observation make them prone to error. In particular, there is always an uncertainty in scoring a performance between two consecutive scores such as 2 and 3 . All the tests have the same weight while some of them might make more contribution to fall. As mentioned before, some tests such as standing on one leg or stepping on a stool can intimidate older people and they refuse to do them. Getting a score of zero in such tests makes the overall score inaccurate.

The system assessment methods are mostly designed for physiological diagnosis purposes. This means that they are capable of recognising the intrinsic factors and causes of balance disorders.

The tools invented for assessing falls in elderly inpatients such as the Stratify tool, need to be short, and easy to conduct as elderly inpatients are very fragile. One of the drawbacks of these methods is that they rely on self-reporting and questionnaires.

Questionnaire-based methods depend heavily on the reports provided by the subjects from their fall experience. There is a high probability that such reports can be biased, inaccurate and possibly incorrect as many subjects may not remember the details of the incident, or may be embarrassed to report truthfully about their experiences. A fall could be caused by extrinsic factors such as a slippery floor and be mistaken as balance impairment. These methods also suffer from the ceiling effect. According to the SAGE dictionary of statistics in data gathering a ceiling effect occurs when the variance in an independent variable cannot be measured above a certain level [102].

Quantitative methods have been proposed for reliable and objective fall risk assessment. Although these methods lead to quantitative, objective results, they can be both expensive and/or difficult to use. Usually, experts and specialists are needed to deploy the specialised equipment. Equipment such as force platforms require significant space and cannot be used everywhere. Operating such equipment often also requires extra time leading to long and tedious tests for older subjects. The inertial sensors, however, have the potential to measure the postural and dynamic balance providing valid and accurate results. The inertial sensors are optimized when they are used together with force platforms. Due to their small size, portability and light weight they can be used anywhere but they must be mounted on the subject's body. This sometimes prevents older people from performing the test comfortably.

The review undertaken in this paper reveals that at present there is no standard or accepted tool for balance and fall risk assessment in older people. Such standards and well-defined and widely accepted tools and methodologies need to be developed.

More rigorous research is also required for extensive study and validation of the quantitative methods deployed in fall risk assessment, capitalising on the learning obtained in qualitative methods, towards a universally proven and accepted methodology for assessing the risk of fall in older people.

Table 1: Summary of Functional Assessment Methods

\begin{tabular}{|c|c|c|c|c|c|}
\hline Tool & Reference & Sensitivity & Specificity & Inter-rate Reliability & Test-retest Reliability \\
\hline Tandem Stance & [21] & $55 \%$ & $94 \%$ & & \\
\hline Tandem Stance & [23] & & & 0.99 & 0.90 \\
\hline One Leg Stance with eyes Open & [23] & & & 0.99 & 0.90 \\
\hline One Leg Stance with eyes Closed & [23] & & & 0.99 & 0.74 \\
\hline Functional Reach Test & [23] & & & 0.96 & 0.86 \\
\hline Functional Reach Test & [21] & $73 \%$ & $88 \%$ & & \\
\hline Sit To Stand Test & {$[23]$} & & & 0.98 & 0.92 \\
\hline CTSIB & [33] & & & 0.99 & \\
\hline TUG & [42] & & & & 0.56 \\
\hline 6MWT & [46] & & & & 0.95 \\
\hline DGI & [48] & $91 \% *$ & $3 \% *$ & & \\
\hline DGI & [47] & & & 0.96 & 0.98 \\
\hline BBS & [52] & $53 \%$ & $96 \%$ & 0.88 & \\
\hline BBS & [54] & & & & 0.8 \\
\hline Tinetti & [58] & $70 \% * *$ & $53 \% * *$ & & \\
\hline Tinetti & [57] & $78 \% * * *$ & $75 \% * * *$ & & \\
\hline $\begin{array}{l}* \text { With cutoff score of } 19 \\
* * \text { With cutoff score of } 36 \\
* * \text { With cutoff score of } 20\end{array}$ & & & & & \\
\hline
\end{tabular}

Table 2: Summary Of system Assessment Methods

\begin{tabular}{|l|c|c|l|l|c|}
\hline Tool & Reference & Sensitivity & Specificity & Inter-rate Reliability & Test-retest Reliability \\
\hline PPA & {$[59]$} & $75 \%$ & & & 0.7 \\
\hline BESTest & {$[62]$} & & & $\mathbf{0 . 9 1}$ & \\
\hline
\end{tabular}


Table 3: summary of System Assessment Methods

\begin{tabular}{|l|c|c|c|c|c|}
\hline Tool & Reference & Sensitivity & Specificity & Inter-rate Reliability & Test-retest Reliability \\
\hline STRATIFY & {$[67]$} & $91 \% *$ & $60 \% *$ & $0.78^{*}$ & \\
\hline Morse & {$[68]$} & $83 \%$ & $68 \%$ & & \\
\hline Morse & {$[64]$} & $78 \%$ & $83 \%$ & 0.96 & 0.86 \\
\hline Downtown Index & {$[70]$} & & & 0.96 & \\
\hline Downtown Index & {$[71]$} & $(81-95) \%$ & $(35-40) \%$ & & \\
\hline BI & \multicolumn{7}{|l}{} \\
\hline With cutoff score of 9 & \multicolumn{5}{l}{} \\
\hline
\end{tabular}

\section{Refrences}

[1]. G. Feder, C. Cryer and S. Donovan, "Guidelines for the Prevention of Falls in People Over 65," British Medical Journal, vol. 321, p. 1007-1011, 2000.

[2]. M. Muray, A. Seireg and S. Sepic, "Normal Postural Stability and Steadiness: Quantitative assessment," The Journal of Bone and Joint Surgery, no. 57A, pp. 510-516, 1975.

[3]. G. Jones, A. Berthoz and B. Segal, "Adaptive modification of the Vestibulo-Occular Reflex By Mental Effort in Darkness," Exp. Brain Research, no. 56, pp. 149-153, 1984.

[4]. G. Tell, D. Lefkowitz, P. Diehr and A. Elster, "Relationship Between Balance and Abnormalities in Cerebral Magnetic Resonance Imaging in Older Adults," Arch Neurology, vol. 55, pp. 73-79, 1998.

[5]. J. Balogun, K. Akindale and J. Nihinola, "Age Related Changes in Balance Performance," Disability and Rehabilitation, vol. 16, pp. 58-62, 1994.

[6]. T. Ikezeo, Y. Asakawa, H. Shima and N. Ichihashi, "Physical Function Screening of Institutionalized Elderly Women to Predict Risk of Falling," Japanese Journal of Physical Fitness and Sports Medicine, vol. 58, no. 5, pp. 489-498, 2009.

[7]. Y. Lajoie and S. Gallagher, "Predicting Falls Within the Elderly Community:Comparison of Postural Sway, Reaction Time, the Berg Balance Scale and the Activities-specific Balance Confidence (ABC) Scale for Comparing Fallers and Non-fallers," Archives of Gerontology and Geriatrics, vol. 38, p. 11-26, 2004.

[8]. M. E. Tinetti and C. S. Williams, "Falls, Injuries Due to Falls, and the Risk of Admission to a Nursing Home," The new England journal of medicine, vol. 337, pp. 1279-1284, 1997.

[9]. J. Close, M. Ellis, R. Hooper, E. Glucksman, S. Jackson and C. Swift, "Prevention of Falls in the Elderly Trial (PROFET): A Randomised Controlled Trial," Lancet, vol. 353, p. 93-97, 1999.

[10]. A. C. Scheffer, N. Van Dijke and T. Van der Hooft, "Fear of Falling: Measurement Strategy, Prevalence, Risk Factors and Consequences Among Older Persons," Age and Aging, vol. 37, pp. 19-24, 2007.

[11]. B. Vellas, F. Cayla, F. de Pemille and J. al Barede, "Prospective Study of Restriction of Activity in Old People After Falls," Age and Aging, vol. 16, no. 3, pp. 189-193, 1987.

[12]. M. E. Tinetti, "Preventing Falls in the Elderly Persons," The New England Journal of Medicine, vol. 348, pp. 42-49, 2003.

[13]. W. Zijlstra and K. Aminian, "Mobility Assessment in Older People: New Possibilities and Challenges," European Journal of Aging, vol. 4, no. 1, pp. 3-12, 2007.

[14]. R. W. Sattin, "Falls Among Older Persons: A Public Health Perspective," Annual Review of Public Health, vol. 13, pp. 489-508, 1992.

[15]. J. A. Stevens, P. S. Corso, E. A. Finkelstein and T. R. Miller, "The Costs of Fatal and Non-fatal Falls Among Older Adults," Injury Prevention, vol. 12, pp. 290-295, 2006.

[16]. D. M. Karantonis, M. R. Narayanan, M. Mathie and N. H. Lovell, "Implementation of a Real-time Human Movement Classifier Using a Triaxial Accelerometer for Ambulatory Monitoring," IEEE Transactions on Information Technology in Biomedicine, vol. 10, no. 1 , pp. 156-167, 2006.

[17]. E. D. de Bruin, A. Hartmann, D. Uebelhart, K. Murer and W. Zijlstra, "Wearable Systems for Monitoring Mobility-related Activities in Older People: A Systematic Review," Clinical Rehabilitation, vol. 22, no. 10-11, pp. 878-895, 2008.

[18]. K. Berg and K. Norman, "Functional Assessment of Balance and Gait," Gait and Balance Disorders, no. 12, pp. 705-723, 1993.

[19]. F. Horak, "Clinical Assessments of Balance Disorders," Gait \& Posture, no. 6, pp. 76-84, 1997.

[20]. A. Khasnis and R. Gokula, "Romberg's Test," Journal of Postgraduate Medicine, vol. 49, no. 2, pp. 169-172, 2003.

[21]. M. A. Murphy, S. L. Olson and E. J. Prots, "Screening for falls in community-dwelling elderly," Journal of ageing and Physical Activity, no. 11, pp. 66-80, 2003.

[22]. Y. Agrawal, J. P. Carey, H. J. Hoffman, D. A. Sklare and M. C. Schubert, "The Modified Romberg Balance Test: Normative Data in US Adults," Otol Neurotol, vol. 8, no. 32, pp. 1309-1311, 2011.

[23]. F. Franchignoni, L. Tesio, M. Martino and C. Ricupero, "Reliability of Four Simple, Quantitative Tests of Balance and Mobility in Healthy Elderly Females," Aging Clinical and Experimental Research, vol. 2, no. 1, pp. 26-31, 1998.

[24]. G. Gehlsen and M. Whaley, "Falls in the Elderly: Part 2, Balance, Strength, and Flexibility," Archs Phys Med Rehabil, no. 71, pp. 739-741, 1990.

[25]. M. Lichtenstein, S. Shields and R. Shiavi, "Comparison of Biomevhanics platform measures and videotaped measures of gait with a clinical mobility scale in elderly women.," Journal of Gerontology: Medical Sciences, vol. 45, pp. 49-54, 1990.

[26]. J. Gill, H. Allum and M. Carpenter, "Trunk Sway Measures of Postural Stability During Clinical Balance Tests: Effects of Age," Journal of Gerontology: Medical Sciences, vol. 56A, no. 7, pp. 438-447, 2001.

[27]. P. Duncan, D. Weiner, J. Chandler and S. Studenski, "Functional Reach: A New Clinical Measure of Balance," Journal of Gerontology, no. 45, pp. 192-197, 1990.

[28]. P. Duncan, S. Studenski and J. Chandler, "Functional Reach: Predictive Validity in a Sample of Elderly Male Veterans," Journal of Gerontology, no. 47, pp. 93-98, 1992.

[29]. A. Behrman, K. Light and S. Flynn, "Is the Functional Reach Test Useful for Identifying Falls Risk Among Individuals with Parkinson's Disease?," Arch of Phys Med Rehabil, no. 83, pp. 538-542, 2002.

[30]. "Simple method for measurement of lower extremity muscle strength," american Journal of Medical, vol. 78, pp. 77-81, 1985.

[31]. P. Macrae, M. Lacrouse and R. Moldavon, "Physical Performance measures that predict faller status in community-dwelling older adults," JOSPT, no. 16, pp. 123-128, 1992.

[32]. A. Shumway-cook and F. Horak, “Assessing the Influence of Sensory Interaction on Balance,” Physical Therapy, vol. 66, no. 10, pp. 1548-1550, 1986. 
[33]. H. Cohen, C. A. Blatchly and L. Gombash, "A Study of the Clinical Test of Sensory Interaction and Balance.," Physical Therapy, vol. 73 , no. 6 , pp. 346-351, 1993 .

[34]. T. K. Crowe, J. C. Deitz, P. K. Richardson and S. W. Atwater, "Interrater Reliability of the Pediatric Clinical Test of Sensory Interaction for Balance," Physical \& Occupational Therapy in Pediatrics, vol. 10, no. 4, pp. 1-27, 1991.

[35]. R. P. Di Fabio and S. Anacker, "Identifying Fallers in Community Living Elders Using a Clinical Test of Sensory Interaction for Balance,” European Journal of Physical Medicine \& Rehabilitation, vol. 6, no. 2, pp. 61-66, 1996.

[36]. A. Yelnik and I. Bonan, "Clinical Tools for Assessing Balance Disorders," Neurophysiol, no. 34, pp. 567-571, 2008.

[37]. S. Mathias, U. Nayak and B. Isaacs, "Balance in Elderly Patients: the " Get Up and Go" Test," Arch. Phys. Med. Rehabil., no. 67, pp. 387-389, 1986.

[38]. A. Shumway-Cook, S. Brauer and M. Woollacott, "Predicting the Probability for Falls in Community-Dwelling Older Adults Using the Timed Up \& Go Test,” Physical Therapy, vol. 80, no. 9, pp. 896-903, 2000.

[39]. L. Lundin-Olsson, L. Nyberg and Y. Gutafson, “Attention, Frailty and Falls: The Effect of Manual Task On Basic Mobility," Journal of American Geraitr, no. 46, pp. 758-761, 1998.

[40]. A. Salarian, C. Zampieri, F. Horak and P. Carlson Kuhta, "Analyzing 180 Degrees Turns Using an Inertial System Reveals Early Signs of Progression of Parkinson's Disease,” in Conf. Proc. IEEE. Eng. Med. Biol. Soc., 2009.

[41]. C. Zampieri, A. Salarian, P. Carlson-Kuhta and K. Aminian, "An Instrumented Timed Up and Go Test Characterizes Gait and Postural Transitions in Untreated Parkinson's Disease,” J. Neurol. NEurosurg. Psychiatry, no. 81, pp. 171-176, 2010.

[42]. K. Rockwood, E. Awalt, D. Carver and C. MacKnight, "Feasibility and measurement properties of the functional reach and the timed up and go tests in the Canadian study of health and aging," Journal of Gerontology, vol. 55, no. 2, pp. 70-73, 2000.

[43]. B. Balke, “A Simple Field Test for the Assessment of Physical Fitness," Rep Civ Aeromed Res Inst US., no. 53, pp. 1 - 8, 1963.

[44]. J. Strijbos, D. Postma, R. Van Altena, F. Gimeno and G. Koeter, "A Comparison between An Outpatient Hospital-based Pulmonary Rehabilitation Program and A Home-care Pulmonary Rehabilitation Program in Patients with COPD. A Follow-up of 18 Months.," Chest, vol. 2, no. 109, pp. 366-72, 1996.

[45]. T. Troosters, R. Gosselink and M. Decramer, "Six Minute Walking Distance in Healthy Elderly Subjects," European Respiratory Journal, vol. 14, no. 2, p. 270-274, 1999.

[46]. N. D. Harada, V. Chiu and A. L. Stewart, "Mobility-related Function in Older adults: Assessment with A 6-minute Walk Test," Archives of Physical Medicine and Rehabilitation, vol. 80, no. 7, pp. 837-841, 1999.

[47]. A. Shumway-Cook, M. Baldwin, N. Polissar and W. Gruber, "Predicting the probability for falls in community-dwelling older adults," Physical Therapy, vol. 77, no. 8, pp. 812-9, 1997.

[48]. T. Herman, N. Inbar-Borovsky, M. Brozgol, N. Giladi and J. M. Hausdorff, "The Dynamic Gait Index in Healthy Older Adults: The Role of Stair Climbing, Fear of Falling and Gender,” Gait \& Posture, vol. 29, no. 2, p. 237-241, 2009.

[49]. L. Vereeck, F. Wuyts, S. Truijen and P. Van de Heyning, "Clinical Assessment of Balance: Normative Data, and Gender and Age Effects.," International Journal of Radiology, vol. 47, no. 2, pp. 67-75, 2008.

[50]. T. J. Stevenson, D. M. Connelly, J. M. Murray, D. Huggett and T. Overend, "Threshold Berg Balance Scale Scores for Gait-Aid Use in Elderly Subjects: A Secondary Analysis," Physiotherapy Canada, pp. 133-140, 2010.

[51]. L. D. B. Thorbahn and R. A. Newton, "Use of the Berg Balance Test to Predict Falls in Elderly Persons," Journal of Physical Therapy, vol. 76, pp. 576-583, 1996.

[52]. N. Maeda, J. Kato and T. Shimada, "Predicting the Probability for Fall Incidence in Stroke Patients Using the Berg Balance Scale," Journal of International Medical Research, vol. 37, no. 3, pp. 697-704, 2009.

[53]. K. Berg, S. Wood-Dauphinee, J. Williams and B. Maki, "Measuring Balance in the Elderly: Validation of an Instrument," Canadian Journal of Public Health, vol. 2, no. 83, pp. 7-11, 1992.

[54]. L. K. Boulgarides, S. M. McGinty, J. A. Willett and C. W. Barnes, "Use of Clinical and Impairment-Based Tests to Predict Falls by Community-Dwelling Older Adults," Physical Therapy, vol. 83, no. 4, pp. 328-339, 2003.

[55]. S. Köpke and G. Meyer, "The Tinetti Test," Zeitschrift für Gerontologie und Geriatrie, no. 39, pp. 288-291, 2006.

[56]. M. E. Tinetti, T. F. Williams and R. Mayewski, "Fall Risk Index for Elderly Patients Based on Number of Chronic Disabilities," The American Journal of Medicine, vol. 80, no. 3, pp. 429-434, 1986.

[57]. D. Kegelmeyer, A. Kloos and K. Thomas, "Reliability and Validity of the Tinetti Mobility Test for Individuals With Parkinson Disease," Journal of the american Therapy Association, no. 87, pp. 1369-1378, 2007.

[58]. M. Raîchea, R. Héberta, F. Princea and H. Corriveaua, "Screening Older Adults at Risk of Falling with the Tinetti Balance Scale," The Lancet, vol. 356, no. 9234, p. 1001-1002, 2000.

[59]. S. R. Lord, H. B. Menz and A. Tiedemann, "A Physiological Profile Approach to Falls Risk Assessment and Prevention," Journal of American Physical Therapy, vol. 83, no. 3, pp. 237-252, 2003.

[60]. S. Lord, J. Ward and A. K. Williams P, "Physiological factors associated with falls in older community-dwelling women," Journal of the American Geriatrics Society, vol. 42, no. 10, pp. 1110-1117, 1994.

[61]. S. R. Lord, R. D. Clark and I. W. Webster, "Physiological Factors Associated with Falls in an Elderly Population," Journal of the American Geriatrics Society, vol. 39, no. 12, pp. 1194-1200, 1991.

[62]. F. Horak, D. Wrisely and J. Frank, "The Balance Evaluation System Test (BESTest) to Differentiate Balance Deficits," Physical Therapy, no. 89, pp. 484-498, 2009.

[63]. F. Franchignoni, F. Horak and M. Godi, "Using Pshychometric Techniques to Improve the Balance Evaluation System'sTest: The Mini BESTest," Archives of Rehab Med.

[64]. J. M. Morse, R. M. Morse and S. J. Tylko, "Development of a Scale to Identify the Fall-Prone Patient / La Revue canadienne du vieillissement," Canadian Journal on Aging, vol. 8, no. 4, pp. 366 - 377, 1989.

[65]. D. Oliver, M. Britton, P. Seed and A. H. Hopper, "Development and Evaluation of Evidence Based Risk Assessment Tool (STRATIFY) to Predict Which Elderly Inpatients Will Fall: Case-Control and Cohort Studies," British Medical Journal, vol. 315, pp. 1049-1053, 1997.

[66]. K. Milisen, N. Staelens, R. Schwendimann, L. D. Paepe, J. Verhaeghe, T. Braes, S. Boonen, W. Pelemans, R. W. Kressing and E. Dejaeger, "Fall Prediction in Inpatients by Bedside Nurses Using the St. Thomas's Risk Assessment Tool in Falling Elderly Inpatients (STRATIFY) Instrument: A Multicenter Study," The American Geriatrics Society, vol. 55, pp. 725-733, 2007.

[67]. A. Papaioannou, W. Parkinson, R. Cook and N. Ferko, "Prediction of Falls Using a Risk Assessment Tool in the Acute Care Setting," BMC Medicine, vol. 2, no. 1, pp. 1-7, 2004.

[68]. M. McCollam, "Evaluation and implementation of a research-based falls assessment innovation," The Nursing Clinics of North America, vol. 30, no. 3, pp. 507-514, 1995.

[69]. J. Downton, "Falls in the Elderly, London," Edward Arnold, vol. 64, no. 80, pp. 128-130, 1993. 
[70]. L. Nyberg and Y. Gustafson, "Using the Downton Index to Predict Those Prone to Falls in Stroke Rehabilitation," Stroke, vol. 27, 1996.

[71]. E. Rosendahl, L. Lundin-Olsson, K. Kallin, J. Jensen, Y. Gustafson and L. Nyberg, "Prediction of Falls among Older People in Residential," Aging Clinical and Experimental Research, vol. 15, no. 2, pp. 142-147, 2003.

[72]. S. Shah, F. Vanclay and B. Cooper, "Improving the sensitivity of the Barthel Index for stroke rehabilitation," Journal of Clinical Epidemiology, vol. 42, no. 8, pp. 703-709, 1989.

[73]. C. Collin, T. D. Wade, S. Davies and V. Horne, "The Barthel ADL Index: A Reliability Study," Disability and Rehabilitation, vol. 10, no. 2, pp. 61-63, 1987.

[74]. W. W. Spirduso, Physical Dimensions of Aging. Human Kinetics, Illinois, USA: Champaign Illinois USA, 1995.

[75]. J. Dornan and G. Fernie, "Visual Input: Its Importance in the Control Postural Sway," Archieves of Physical Medicine and Rehabilitation, vol. 59, pp. 586-591, 1978.

[76]. J. Brocklehurst and D. Robertson, "Clinical Correlates of Sway in Old Age: Sensory Modalities," Age and Ageing, vol. 11, pp. 1$10,1982$.

[77]. J. Sheldon, "The Effect of Age on the Control of Sway," Gerontological Clinica, vol. 5, pp. 129-138, 1963.

[78]. P. Era and E. Heikkinen, "Postural Sway During Standing and Unexpected Disturbance of Balance in Random Samples of Men of Different Ages," Journal of Gerontology, vol. 40, no. 3, pp. 287-295, 1985.

[79]. B. Maki and P. Holliday, "A Prospective study of Postural Balance and Risk of Falling in an Ambulatory and Independent Elderly Population," Journal of Gerontology: Medical Sciences, vol. 49, no. 2, pp. 72-84, 1994.

[80]. M. Davidson, "Effects of Lumbar Extensor Fatigue and Fatigue Rate on Postural Sway," European Journal of Applied Physiology, vol. 93, pp. 183-189, 2004.

[81]. H. Thyssen, J. Brynskov, E. Jansen and J. Münster-Swendsen, "Normal Ranges in Reproducibility for Quantitative Romberg's Test. Acta Neurol Scand," Acta Neurol Scand, vol. 1, no. 66, pp. 100-104, 1982

[82]. E. Dean, C. Griffith and A. Murray, "Stability of the Human Body Investigation by Sway Magnetometry," Journal of Medical Engineering \&Technology, no. 10, pp. 126-130, 1986.

[83]. B. Wright, “A Simple Ataxia-Meter,” Journal of Physiology, no. 218, pp. 27P-28P, 1971.

[84]. G. Fernie and P. Holiday, "Postural Sway in Amputees and Normal Subjects," The Journal of Bone and Joint Surgery, vol. A, no. 60, pp. 895-898, 1978

[85]. U. S. L. Nayak, "Comparison of the Wright Ataxiameter and the Kistler Force Platform in the Measurement of Sway," Journal of Biomedical Engineering, vol. 9, no. 4, pp. 302-204, 1987.

[86]. G. Fernie and P. Holiday, "Postural Sway in Amputees and Normal Subjects," The Journal of Bone and Joint Surgery, vol. A, no. 60, pp. 895-898, 1978.

[87]. J. Browne and N. O'Hare, “A Review of the Different Methods for Assessing Standing Balance,” Physiotherapy, vol. 9, no. 87, pp. 489-495, 2001.

[88]. W. BM, “A simple mechanical ataxia-meter," The Journal of Physiology, vol. 218, pp. 27P-28P, 1977.

[89]. J. C. Brocklehurst, D. Robertson and P. James-Groom, "Skeletal Deformities in the Elderly and Their Effect on Postural Sway," Journal of the American Geriatrics Society, vol. 30, no. 18, p. 534-538, 1982.

[90]. J. Swanenburg, E. D. de Bruin, D. Uebelhart and T. Mulder, "Falls Prediction in Elderly People: A 1-year Prospective Study," Gait \& Posture, vol. 31, no. 3, pp. 317-321, 2009.

[91]. A. Zijlstra, T. Ufkes, D. Skelton, L. Lundin-Olsson and W. Zijlstra, "Do Dual Tasks Have an Added Value Over Single Tasks for Balance Assessment in Fall Prevention Programs? A Mini-Review,” Gerontology, vol. 54, p. 40-49, 2008.

[92]. P. S. Haibach, S. M. Slobounov and K. M. Newell, "The Potential Application of a Virtual Moving Environment for Assesing Falls in Elderly Adults," Gait and Posture, vol. 27, pp. 303-308, 2008.

[93]. D. C. Kerrigan, L. W. Lee, T. J. Nieto, J. D. Markman, J. J. Collins and P. O. Riley, "Kinetic Alterations Independent of Walking Speed in Elderly Fallers," Archives of Physical Medicine and Rehabilitation, vol. 81, no. 6, p. 730-735, 2000.

[94]. J. Verghese, R. Holtzer and R. B. Lipton, "Quantitative Gait Markers and Incident Fall Risk in older adults," Journal of Gerontology: Medical Sciences, vol. 8, p. 896-901, 2009.

[95]. D. C. Kerrigan, M. K. Todd, U. D. Croce and L. A. Lipsitz, "Biomechanical Gait Alterations Independent of Speed in the Healthy Elderly: Evidence for Specific Limiting Impairments," Archive of Physical Medicine and Rehabilitation, vol. 79, pp. 317-322, 1998.

[96]. D. A. Winter, A. E. Patla, J. S. Frank and S. E. Walt, "Biomechanical Walking Pattern Changes in the Fit and Healthy Elderly," Physical therapy, vol. 70, pp. 340-347, 1990.

[97]. B. Najafi, K. Aminian, C. Bula, G. Ruggieri and P. Robert, "Falling Risk Evaluation in Elderly Using Miniature Gyroscope: Relation Between Gait and Risk of Falling," in ISPGR2001, Netherlands, 2001.

[98]. B. Najafi, K. Aminian, F. Loew, Y. Blanc and P. A. Robert, "Measurement of Stand-sit and Sit-stand Transitions Using a Miniature Gyroscope and Its Application in Fall Risk Evaluation in the Elderly," IEEE Transactions on Biomedical Engineering, vol. 49, no. 8, pp. $843-851,2002$.

[99]. H. J. Yack and R. C. Berger, "Dynamic Stability in the Elderly: Identifying a Possible Measure," The Journal of Gerontology, vol. 48, no. 5, pp. 225-230, 1993.

[100]. H. B. Menz, S. R. Lord and R. C. Fitzpatrick, “Acceleration Patterns of the Head and Pelvis When Walking Are Associated with Risk of Falling in Community-dwelling Older People," The Journal of Gerontology, vol. 28, no. 5, pp. 446-452, 2003.

[101]. B. Mariani, C. Hokovec, S. Roghat, C. Bula, J. Penders and K. Aminian, "3D Gait Assessment in Young and Elderly Subjects Using Foot-worn Inertial Sensors,” Journal of Biomechanics, vol. 43, no. 15, p. 2999-3006, 2010.

[102]. D. Cramer and D. Howitt, The Sage dictionary of statistics: a practical resource for students in the social sciences, SAGE publication, 2005.

[103]. A. S. Robbins, L. Z. Rubenstein and K. R. Josephson, "Predictors of Falls Among Elderly People: Results of Two Population-Based Studies," Archive of Internal Medicine, vol. 149, no. 7, pp. 1628-1633, 1989.

[104]. P. G. Macrae, J. F. Schnelle and S. F. Simmons, "Physical Activity Levels of Ambulatory Nursing Home Residents," Journal of Aging and Physical Activity, vol. 4, p. 264-278, 1996. 
Fall Risk

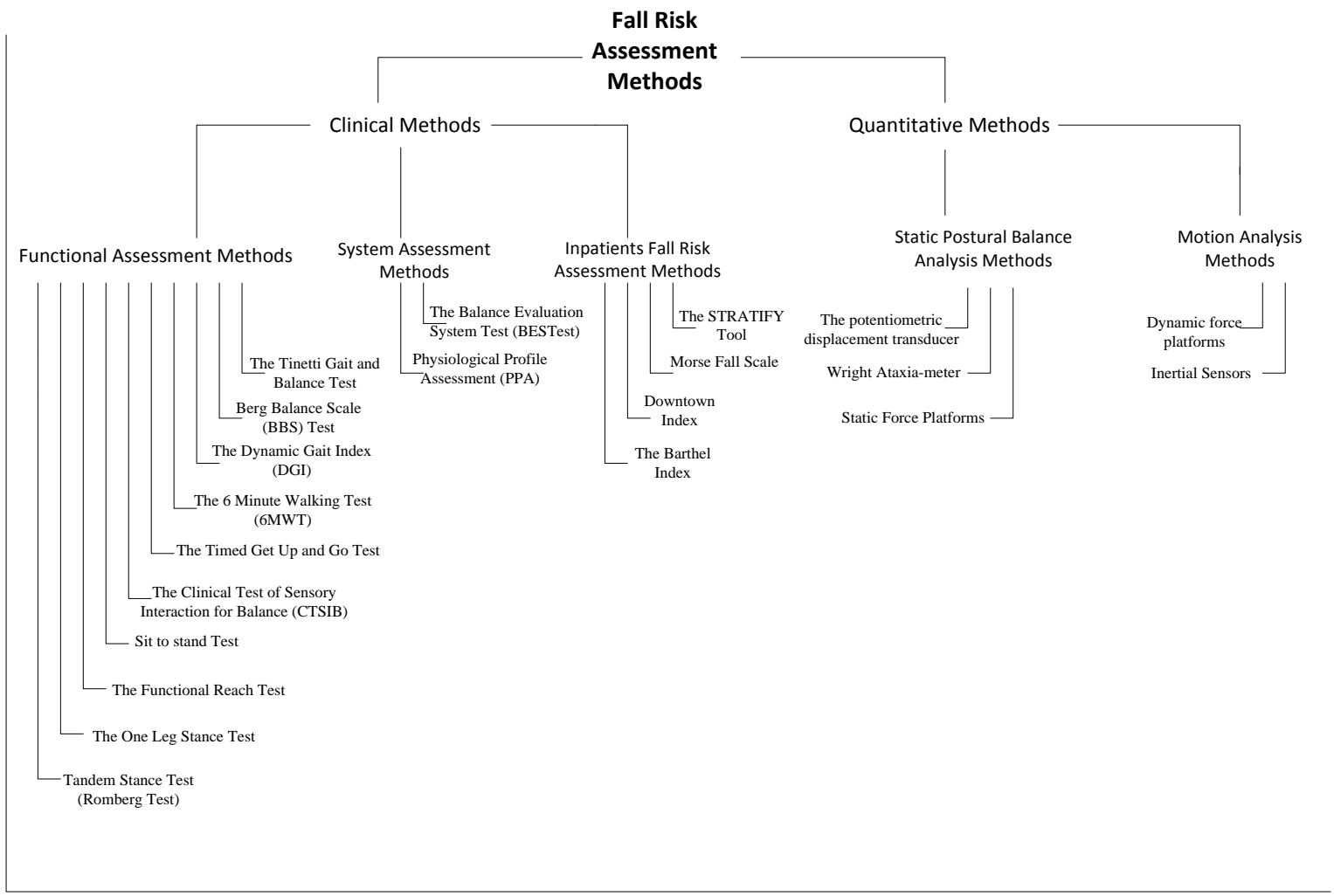

